\title{
Role of Mitotane in Adrenocortical Carcinoma - Review and State of the art
}

\author{
Rosa Maria Paragliola, ${ }^{1,2}$ Francesco Torino, ${ }^{3}$ Giampaolo Papi, ${ }^{1}$ Pietro Locantore, ${ }^{1,2}$ Alfredo Pontecorvi ${ }^{1,2}$ \\ and Salvatore Maria Corsello, ${ }^{1,2}$ \\ 1. Unit of Endocrinology, Università Cattolica del Sacro Cuore, Rome, Italy; 2. Fondazione Policlinico Universitario Agostino Gemelli, Rome, Italy; \\ 3. Department of Systems Medicine, Medical Oncology, University of Rome Teor Vergata, Rome, Italy.
}

A drenocortical carcinoma (ACC) is a rare and aggressive endocrine tumour deriving from the adrenal cortex. A correct therapeutic strategy requires a multidisciplinary approach between endocrinologist, surgeon and oncologist. Surgery is the mainstay treatment in ACC while mitotane, deriving from the insecticide dichloro-diphenyl-trichloro-ethane, is the main base of the medical treatment of ACC in consideration of its adrenocytolitic activity. However, the use of mitotane as adjuvant therapy is still controversial, also in consideration of the retrospective nature of several studies. A prospective randomised trial (ADIUVO), recruiting patients with low-intermediate risk of recurrence, is evaluating the utility of adjuvant treatment with mitotane in this setting. The therapeutic response is observed with plasma levels of mitotane $>14 \mathrm{mg} / \mathrm{L}$. However, the major difficulty in the management of mitotane treatment is related to side effects and to the risk of toxicity, which is related to plasmatic levels $>20 \mathrm{mg} / \mathrm{L}$, that is considered the upper limit of the therapeutic window. Mitotane therapy results in adrenal insufficiency, and glucocorticoid replacement therapy has to be administered at higher doses than those used in other aetiologies of primary adrenal insufficiency. Furthermore, other endocrine side effects related to mitotane should be considered, in particular on thyroid hormone and testosterone metabolism. Waiting for new medical strategies on molecular targets, it will be mandatory to optimise the current knowledge by prospective trials and, in consideration of the rarity of the disease, collaborative studies between endocrinologists and oncologists are necessary.

\section{Keywords}

Mitotane, adrenocortical carcinoma, mitotanemia, Cushing's syndrome

Disclosure: Rosa Maria Paragliola, Francesco Torino, Giampaolo Papi, Pietro Locantore, Alfredo Pontecorvi and Salvatore Maria Corsello have no relevant conflicts of interest to declare.

Review Process: Double-blind peer review.

Authorship: All named authors meet the criteria of the International Committee of Medical Journal Editors for authorship for this manuscript, take responsibility for the integrity of the work as a whole and have given final approval for the version to be published.

open Access: This article is published under the Creative Commons Attribution Noncommercial License, which permits any non-commercial use, distribution, adaptation and reproduction provided the original author(s) and source are given appropriate credit. (C) The Authors 2018.

Received: 9 May 2018

Accepted: 29 June 2018

Citation: European Endocrinology, 2018;14(2):62-6

Corresponding Author: Salvatore M Corsello,

Endocrinology, Università Cattolica del Sacro

Cuore, Largo Francesco Vito 1, I-00167, Rome,

Italy. E: salvatore.corsello@unicatt.it

Support: No funding has been received in the publication of this article.
Adrenocortical carcinoma (ACC) is a rare endocrine tumour deriving from the adrenal cortex. Its estimated incidence is one per million/year, ${ }^{1}$ with an increased incidence in the first and fourth to fifth decades of life and with a higher prevalence in females (up to 60\%). ${ }^{2}$ Prognosis is generally poor and 5 -year overall survival is only between $15-44 \% .^{3-5}$ However, the outcome is very heterogeneous and difficult to predict, due to the great variability in clinical presentation, which ranges from tumours with an indolent clinical course to aggressive tumours with fatal outcome.

The stage of the disease at diagnosis is a key prognostic factor in ACC with an expected 5-year survival of $80 \%$ in patients with stage I, while stage IV is associated to a 5 -year survival of $13 \%{ }^{6}$ Furthermore, about two-thirds of patients with localised disease have recurrence, which often requires systemic chemotherapy. ${ }^{7}$

Correct therapeutic strategy requires a multidisciplinary approach. Surgery is the mainstay treatment in ACC and brings the best possibility for prolonged survival and potential cure in localised disease, while it is often not feasible in advanced disease. Inoperable or metastatic disease may benefit from systemic treatment, which is unfortunately limited to mitotane, a dichloro-diphenyl-trichloro-ethane (DDT) derivative. Moreover, its use as adjuvant therapy is still controversial. Herein we review the pharmacological and clinical features of mitotane in patients affected by ACC.

\section{Mitotane - mechanisms of action}

Mitotane is an adrenocytolytic drug which represents the only therapeutic option approved by the US Food and Drug Administration and the European Medicine Executive Agency for the treatment of ACC. ${ }^{8}$ It derives from the insecticide DDT and, approximately 70 years ago, it was recognised as an agent that selectively damages adrenocortical tissue. ${ }^{9}$ Since 1959, mitotane has been used for the treatment of inoperable ACC, but its role in prolonging survival is still debated. ${ }^{10}$

Mitotane sensitivity is different between species. The toxic effects have been widely studied in dogs, a particularly sensitive species. In humans, high mitotane doses cause adrenal atrophy, while rats, mice, rabbits and monkeys are relatively unresponsive. 9 At microscopic level, mitotane can cause the destruction of both the zona fasciculata and reticularis; however, its adrenocytolytic activity saves the zona glomerulosa, with selective loss of steroid secretion 
limited to hormones which are under the control of adrenocorticotropic hormone (ACTH) secretion. ${ }^{11}$ In dogs, mitotane administration destroys the zona glomerulosa in about $6-10 \%$ of cases or decreases the aldosterone secretory reserve. ${ }^{12,13}$

At the cellular level, the most important effect of the drug can be observed in mitochondria, which in turn presents unique characteristics in steroidogenic tissue and plays a crucial role in both the evolution to malignancy and apoptosis. ${ }^{9}$ To clarify the molecular mechanisms of mitotane action, metabolomic and lipidomic techniques have been employed on human ACC cell line..$^{14}$ This study shows that mitotane exerts a critical role in the dysfunction of mitochondria-associated membranes. In particular, mitotane induces an impairment of respiratory chain, decreasing aspartate and concomitantly increasing glutamate content in a time- and concentration-dependent manner. ${ }^{14}$

Furthemore mitotane reduces the phosphatidylserine phosphatidylethanolamine ratio, causing a dysfunction of phosphatidylserine decarboxylase located in mitochondria-associated membranes. ${ }^{14} \mathrm{~A}$ hydroxylation occurring in the liver produces mitotane metabolites ( $0^{\prime}, \mathrm{p}$-DDA and o', $\mathrm{p}$-DDE, respectively), producing $o^{\prime}, p-D D A$, the active form of the drug. ${ }^{15}$

Several mechanisms sustain the inhibitory effects of mitotane on steroidogenesis. In vitro studies on ACC cell line (NCl-H295R) show a decrease in cell viability and an increase in caspase-3 and -7 activities. Furthermore, mitotane causes decreased mRNA levels of two cytochromes p450 (CYP11A1 and CYP17A1), encoding proteins involved in cortisol and dehydroepiandrosterone sulphate biosynthesis. ${ }^{16}$ The expression of both transforming growth factor beta1 gene and protein kinase-A regulatory subunit PRKAR1A is reduced after mitotane exposure. ${ }^{16}$ The inhibition of CYP11A1 described in vitro can be related to a decreased conversion of cholesterol to pregnenolone, which in turn could explain cases of hypercholesterolemia that can be seen in patients under mitotane. ${ }^{17}$ However, previous studies suggest that hypercholesterolemia might be dependent on an increased cholesterol synthesis related to the mitotane-induced up-regulation of 3-hydroxy3-methyl-glutaryl-coenzyme A-reductase activity. ${ }^{18,19}$

Studies addressing the impact of mitotane on adrenal steroidogenesis

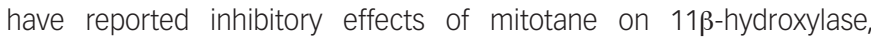
$3 \beta$-hydroxysteroid dehydrogenase, and 18-hydroxylase activities.0,21 In contrast, another study did not find any change in 11-deoxycortisol metabolite excretion in humans, suggesting that a significant change in 11 1 -hydroxylase (CYP11B1) enzymatic activity is highly unlikely. ${ }^{17}$ A recent study on ACC model cell line demonstrates that mitotaneadrenocytolytic activity and down-regulation of steroidogenesis can be due to lipid-induced endoplasmic reticulum-stress through inhibition of activity of sterol-0-acyltransferase-1.22

In addition to its inhibitory effects on multiple enzymatic steps of adrenocortical steroid biosynthesis, mitotane increases cortisol binding globulin (CBG) and sex hormone binding globulin (SHBG) ${ }^{23}$ The increased SHBG and CBG gene expression and liver production is associated with mechanisms requiring the presence of oestrogen receptor alpha. ${ }^{24}$ Furthermore, mitotane increases thyroxine-binding globulin and competes with thyroxine for thyroxine-binding globulin sites. ${ }^{23}$ Finally, as mitotane is an inducer of hepatic cytochrome CYP34A, it can interfere with the metabolism of various drugs, including antihypertensives, statins, antibiotics, chemotherapeutic agents and coumarin-like anticoagulants. Patients under the latter drugs should be closely monitored for a change in anticoagulant dose requirements. ${ }^{25,26}$

\section{Mitotane - clinical efficacy, dosage and concentration}

Currently, mitotane is the only approved single agent for the treatment of patients affected by ACC. Several studies on efficacy of mitotane have reported conflicting results, probably related to possible biases, including different dosages of the drug and the retrospective nature of the studies. ${ }^{27}$ One of the most important contributions to this field is a retrospective analysis involving 177 patients from Italy and Germany treated with mitotane after radical surgery, showing that recurrence free survival (RFS) was significantly prolonged in the mitotane group (42 months) compared with the control groups (10 months and 25 months). Furthermore, overall survival seems to be increased in patients receiving adjuvant mitotane. ${ }^{28}$ However, this study presents some limitations related to its retrospective analysis (bias in selection, diagnosis and follow-up). The authors emphasised that, to reduce the section bias, they included all consecutive eligible patients independently of the characteristics of the patients (for mitotane-treated group and control group 1, from Italian centres). Furthermore, control group 2 was derived from a large nationwide registry of patients with ACC in Germany, reasonably representative of all patients with resected ACC during the study period. ${ }^{28}$

A large single-centre analysis has been offered by Else et al. ${ }^{29}$ including 391 adult patients with the diagnosis of ACC. In this study, adjuvant therapy with mitotane improves RFS (but not overall survival), especially if combined with radiation therapy. Radiation therapy results very effective in preventing local recurrence.

A recent retrospective series analysed a group of patients $(\mathrm{N}=36)$ with metastatic ACC treated with mitotane as single agent at the Memorial Sloan Kettering Cancer Center from 1989-2015.30 In this series, three patients (8\%) achieved a complete response, one patient a partial response (3\%), and one patient (3\%) obtained a stable disease after slow disease progression prior to initiation of therapy (durable for 6 months). However, in consideration of the retrospective nature, the results of these studies have to be carefully evaluated.

Presently, a multicentric international clinical trial (Efficacy of Adjuvant Mitotane Treatment, ADIUVO) evaluating the efficacy of adjuvant mitotane $^{31}$ in patients with low-intermediate risk of recurrence is ongoing. ${ }^{32}$ The primary endpoint of this trial is to evaluate the efficacy of adjuvant mitotane treatment versus non-treatment in improving the RFS in patients al low-intermediate risk. Secondary endpoints will be focused in evaluating the overall survival, the quality of life, the assessment of toxicity and the efficacy of mitotane administration on subgroups of patients according to the type of hormone secretion, stage of disease and histopathological characteristics. ${ }^{32}$

Waiting for the results from ADIUVO trial, the recent European Society of Medical Oncology (ESMO) guidelines on treatment of ACC recommend to use adjuvant mitotane treatment in stage III patients with potential residual disease (R1 or Rx resection status) and $\mathrm{Ki}-67>10 \% \cdot{ }^{33}$ For patients in stages I or II, RO resection and $\mathrm{Ki}-67<10 \%$, the quality of evidence to recommend for or against the adjuvant use of mitotane is considered very low. ${ }^{27,33}$ The National Comprehensive Cancer Network (NCCN) guidelines for adrenal gland tumours, suggest adjuvant therapy with mitotane in patients with localised disease with high risk for local recurrence after surgery (positive margins, rupture of the capsule, large size and high grade). ${ }^{34}$ The more recent European Society of Endocrinology Clinical Practice Guidelines on the Management of Adrenocortical Carcinoma, published in collaboration with the European Network for the Study of Adrenal Tumors, suggest adjuvant mitotane treatment in patients without macroscopic residual 
tumour after surgery, but with high risk of recurrence, ${ }^{35}$ as suggested by ESMO guidelines. On the other hand, there is no advice for or against adjuvant therapy for patients with low-moderate risk of recurrence and the therapy should be discussed individually for each patient.

Mitotane is available as $500 \mathrm{mg}$ tablets, which should be taken with a glass of water during meals containing fat-rich foods. The total daily dose may be titrated in two or three doses. ${ }^{31}$ In adult patients, treatment should be started with a "low-dose regimen" (1 gr/day to increase to $3 \mathrm{gr} /$ day in 2 weeks) or with a "high dose regimen" (1.5 gr/day to increase to 6 gr/day in 4-6 days), ${ }^{10}$ until reaching a plasma mitotane of $14-20 \mathrm{mg} / \mathrm{L} .{ }^{31}$ These levels are considered an "ideal" therapeutic window that assures the efficacy of the drug with acceptable safety.

There is clear evidence of a correlation between mitotane plasma levels and antineoplastic efficacy. In particular, with plasma levels above $14 \mathrm{mg} / \mathrm{L}$ an objective response rate up to $55-66 \%$ has been achieved, whereas lower levels predicted lower efficacy. ${ }^{15,36}$ Other studies report lower percentage of objective tumour response (19\%), but also in this case, plasma mitotane concentration was higher in responders and more responders were found in the group of patients with mitotane plasma levels of $14 \mathrm{mg} / \mathrm{L}$ or greater. ${ }^{37}$

The anti-neoplastic effect of mitotane appears to be correlated with plasma levels. In fact, mitotane concentrations $\geq 14 \mathrm{mg} / \mathrm{L}$ are associated with a prolonged RFS in patients following macroscopically radical surgery. ${ }^{38,39}$ Therefore, the major goal during mitotane therapy is to have plasma levels above $14 \mathrm{mg} / \mathrm{L}$ and below $20 \mathrm{mg} / \mathrm{L}$, that is considered the upper limit of the therapeutic window to avoid toxicities. ${ }^{36}$ At daily low dose of mitotane, the target plasma concentration is usually reached within a period of 3-5 months (total mitotane dose of 283-387 g). ${ }^{40}$

Mitotane plasma levels should be monitored at frequent intervals (approximately every 2 weeks) until the therapeutic window has been reached. ${ }^{.1}$ The monitoring should be more frequent if a higher starting dose has been used. Once the maintenance dose has been reached, mitotane levels should be monitored every month. In overweight patients, it is important to consider that fat tissue can act as a reservoir for mitotane, ${ }^{41}$ causing a prolonged half-life and potential accumulation of mitotane. ${ }^{31}$

As reported in many retrospective studies, the difficulty in the management of this therapy is often related to the requirement of high doses of mitotane to maintain a therapeutic range capable of inducing a beneficial outcome in patients with ACC. ${ }^{42}$ However, starting high-doses of mitotane in monotherapy (4-6 g per day) seems to induce prolonged survival in patients, without significant increases in toxicity if compared with low-dose regimen (2-3 g per day). ${ }^{43}$

In the clinical practice, mitotane plasma concentrations are determined by high-performance liquid chromatography at Atlanbio laboratory (Saint-Nazaire, France) using the Lysosafe service offered by HRA Pharma (Paris, France). ${ }^{44}$ Mitotane plasma levels show short-term variability during the day; consequently, random sampling could yield incidentally high levels. For this reason, trough sampling is considered the standard management in monitoring mitotane levels and early morning appears the optimum time to assess trough levels. ${ }^{45}$

In presence of serious adverse reactions, such as neurotoxicity and acute hepatic impariment, treatment with mitotane should be stopped. Liver function tests should be performed periodically (roughy every 3 months²) during the treatment. Transaminases' increase is encoutered in the vast majority of patients treated with mitotane and in most cases no action is required. ${ }^{46}$ However, with rapidly rising levels of aspartate aminotransferase and alanine aminotransferase, or levels greater than 3-4 fold the normal range, mitotane therapy should be temporarily stopped. Furthermore, evaluation for mitotane induced toxicity or other liver pathologies should be started. ${ }^{2}$ Patients can also experience mild to moderate elevation of alkaline phosphatase and gamma-glutamyltransferase, but these effects are very common and no action is required. ${ }^{2}$

Most patients also report gastrointestinal disturbances (nausea, vomiting, diarrhoea, epigastric discomfort). ${ }^{42}$ However, gastrointestinal and hepatic toxicities are acceptable if rather low doses of mitotane (up to $6 \mathrm{~g}$ daily) are used. ${ }^{42}$ On the other hand, mitotane is mainly metabolised by the liver and levels are expected to be increased in presence of a severe hepatic impairment. Therefore, mitotane cannot be prescribed in the presence of severe hepatic impairment.

Common neurological disturbances include ataxia, paraesthesia, vertigo, dizziness, headache and polyneuropathy. The symptoms are more frequent when plasma levels of the drug are above $20 \mathrm{mg} / \mathrm{L} .{ }^{47}$ Pape and colleagues recently reported a case of metabolic encephalopathy diagnosed in a 25-year-old woman treated with high-dose mitotane (4 g per day). ${ }^{48} \mathrm{In}$ case of mild toxicity, the dosage should be reduced until the maximum tolerated dose has been reached. Blood and lymphatic system disorders have also been reported (leukopenia, anaemia, thrombocytopenia and prolonged bleeding time). ${ }^{31}$ Gynecomastia, skin rashes and asthenia have also been reported. ${ }^{42}$ The use of mitotane should be carefully evaluated in cases of mild or moderate renal impairment, as it is not recommended in presence of a severe kidney injury. ${ }^{31}$

The use of mitotane in paediatric populations is limited and its effectiveness remains to be recognised. In literature, the experience concerning the use of mitotane in paediatric populations is limited to case reports or small numbers of patients. The posology has not been well established, even if it seems to be equivalent to that of an adult population corrected for body mass. ${ }^{31}$ Treatment should be started at $1.5-3.5 \mathrm{~g} / \mathrm{m}^{2} /$ day and then increased until reaching $4 \mathrm{~g} / \mathrm{m}^{2} /$ day. However, even in adults, a wide range of doses is used depending on the individual, and the therapeutic range is between $14-20 \mathrm{mg} / \mathrm{L}$. Mitotane levels of more than $20 \mathrm{mg} / \mathrm{L}$ are associated, also in children, with neurological toxicity. ${ }^{49}$ The recovery from toxicity in children requires a long-term follow-up. Some authors suggest the use of mitotane in cases of poor prognosis, such as in p53 mutations carriers. ${ }^{49}$ However, further studies will be necessary to establish the effectiveness and the safety of mitotane in paediatric population.

\section{Adrenal insufficiency}

Despite its widespread use in ACC, knowledge about the effect of mitotane on steroidogenesis, is limited. However, mitotane therapy results in adrenal insufficiency and some clinicians prefer to initiate cortisol replacement concomitantly with mitotane. Furthermore, it is well established in clinical practice that glucocorticoid replacement therapy has to be administered at higher doses than those used in other aetiologies of primary adrenal insufficiency to avoid adrenal crisis. ${ }^{50}$ The evidence of alterations in cortisol metabolism, related to impaired hepatic enzyme activity, was documented shortly after the introduction of mitotane for the treatment of ACC and an increased urinary excretion of $6 \beta$-hydroxycortisol in guinea pigs and humans has been reported. .1,52 $^{2}$ The induction of drug-metabolising liver enzymes has been suspected on the basis of the increased metabolism of pentobarbital, ${ }^{29}$ hexobarbital and cortiso ${ }^{30}$ during mitotane treatment. The effects of mitotane on in vivo steroidogenesis have been recently investigated with urinary steroid 
metabolomic analysis in 24-hour urine samples. ${ }^{17}$ The authors found an increase in the excretion of $6 \beta$-hydroxycortisol over cortisol, indicative of a strong induction of the cytochrome P4503A4. This mechanism is responsible for the rapid inactivation of more than $50 \%$ of administered hydrocortisone. For this reason, the authors suggest that glucocorticoid replacement therapy should be started and maintained with at least double the dose normally used in primary adrenal insufficiency (40-50 mg of hydrocortisone or $75 \mathrm{mg}$ of cortisone acetate). Dexamethasone should be avoided because of inducing effect of mitotane on P4503A4, which can result in rapid inactivation of dexamethasone. ${ }^{17}$

The possibility of the use of modified release hydrocortisone in mitotane treated patients has been evaluated..$^{53}$ However, in patients treated with mitotane, modified release hydrocortisone resulted in much lower total and free cortisol levels than immediate release hydrocortisone at the same dose. ${ }^{53}$ Furthermore, modified release hydrocortisone in patients with ACC does not guarantee the physiological morning free cortisol peak. In addition, considering the increased CYP3A4 activity induced by mitotane, the once daily regimen might not be enough to assure a sufficient glucocorticoid replacement therapy over the whole day. ${ }^{53}$

In the clinical practice, the interpretation of a biochemical test is crucial both for the diagnosis of hypoadrenalism and for monitoring to assess therapy. ${ }^{54}$ Additionally, in some cases, concomitant Cushing's syndrome associated to ACC should be considered. However, in mitotane-treated patients, corticosteroids and ACTH values can be impaired by the interference of this drug. Serum cortisol cannot be considered a valid parameter, since mitotane increases CBG which artificially raises total cortisol. ${ }^{23}$ The utility of the assessment of serum free cortisol levels in mitotane-treated patients has been evaluated. ${ }^{55}$ However, a more useful method can be represented by salivary cortisol, which is not affected by CBG alterations and reflects the serum-free cortisol status. In particular, salivary cortisol measured by tandem-mass spectrometry represents a selective method, which allows the exclusion of potentially-interfering cortisol analogues accumulating in mitotane-treated patient. ${ }^{56} \mathrm{~A}$ recent study proposed the utility of hair cortisol measurement in mitotanetreated ACC patients for the long-term monitoring of hydrocortisone treatment. ${ }^{57}$ Moreover, mitotane can cause falsely elevated urinary free cortisol levels due to altered steroid clearance. ${ }^{23}$

ACTH levels can vary widely in different patients. In particular, ACTH is a pulsatile indirect parameter of circulating corticosteroids and may remain persistently suppressed in patients with concomitant longstanding hypercortisolism. Even if the more consistent consequence of mitotane on adrenal function is an increase of ACTH levels related to hypoadrenalism, studies in vitro have also documented a direct inhibitory effect of mitotane at pituitary level and hypothalamic levels. ${ }^{58}$ The effects are a reduction of pituitary function and cell survival in mouse models and in culture of human ACTH secreting pituitary adenomas, as well as a reduction of $\mathrm{ACTH}$ secretion related to the lack of stimulatory effects of corticotropin-releasing hormone. ${ }^{58}$ This hypothesis has been confirmed in clinical studies. A prospective evaluation of 16 patients on adjuvant treatment with mitotane after radical surgical resection of ACC, showed lower ACTH levels in patients with ACC compared with patients with primary adrenal insufficiency, both in basal conditions and after corticotropin-releasing hormone stimulation, confirming the inhibitory effect on ACTH secretion at the pituitary levels..$^{59}$ Mitotane also acts on the hypothalamus-pituitary-thyroid axis, impairing thyroid-stimulating hormone expression and thyrotropin-releasing hormone responsivity in experimental models. ${ }^{60}$ In clinical studies, a marked reduction in free thyroxine (FT4) levels (inversely correlated with mitotane concentrations) has been observed. Interestingly, thyroid-stimulating hormone did not change accordingly. The explanation can be attributed to a possible "central hypothyroidism" or alternatively to an impairment of desiodase activity which changes FT4/ free tri-iodothyronine (FT3) ratio. However, a thyroid function test should be performed during mitotane treatment and some patients may require levothyroxine (LT4) replacement therapy. ${ }^{42}$

In males, hypogonadism can be detected. This finding can be related both to the above-mentioned effect of mitotane on SHBG synthesis and to a reduction of testosterone secretion by testes or induced by lack of pituitary luteinising hormone increase. ${ }^{42}$ The mitotane effect on luteinising hormone seems to be related to its weak "oestrogen-like activity", as demonstrated by the slight hyperprolactinemia detected in some patients. ${ }^{42}$ Furthermore, in female patients gonadal function seems to be mostly preserved. In males, strong inhibition of systemic $5 \alpha$-reductase activity has also been documented, indicated by a significant decrease in $5 \alpha$-reduced steroids. ${ }^{17}$ This is concordant with the clinical observation of the possible relative inefficiency of testosterone replacement in mitotane-treated men. ${ }^{17}$

\section{Mitotane plus chemotherapy}

Patients diagnosed with advanced ACC and low tumour burden seem to benefit from mitotane monotherapy, while in patients with metastatic disease at the time of diagnosis, mitotane monotherapy might not be the treatment of choice. In these patients, the opportunity to start a treatment combining mitotane with cytotoxic drugs should be evaluated, as suggested by a recent cohort study. ${ }^{39}$ Unfavourable prognostic parameters, such as high tumour burden, uncontrolled symptoms, fast growing tumour and high proliferative index, are important factors suggesting a more sustained therapeutic approach.

Several cytotoxic agents have been used alone or in combination with mitotane in the treatment of advanced ACC.61 The aim of chemotherapy associated with mitotane is related to the ability of mitotane to overcome the drug resistance induced by P-glycoprotein which is widely expressed in ACC. ${ }^{27,62}$ The efficacy of the combination of streptozocin with mitotane (1-4 gr/day) has been evaluated in a phase II study, involving 40 patients affected by ACC. Complete or partial response was obtained in $36.4 \%$ of patients and the presence of distant metastases at diagnosis was identified as a poor prognostic factor. ${ }^{63}$

Subsequently, a large prospective phase II trial evaluated the efficiency of polichemotherapy (etoposide-doxorubicin-cisplatin [EDP]) in combination with mitotane (4 gr/day per os). ${ }^{64}$ In this trial, the overall response rate was $48.6 \%$ and 5 out of 72 patients obtained a complete response. ${ }^{64}$ Interestingly, in this group, androgen and glucocorticoid secretions were associated with long survival and poor prognosis respectively. Furthermore, the surgical resection of residual disease after chemotherapy led to a more favourable outcome. The most frequent toxicity was haematologic (leukopenia), while the most common extra-haematologic side effects were gastrointestinal, neurologic, asthaenia and myalgias. This study confirmed that the therapeutic activity of EDP plus mitotane seems to be higher (in terms of response rate and survival) than that of either mitotane alone or other chemotherapy regimens associated or not to mitotane. ${ }^{64}$ A larger randomised study conducted by Fassnacht et al., and including 304 patients, confirmed a significantly higher response and longer median progression-free survival in the group of patients treated with mitotane plus EDP compared to mitotane plus streptozocin..$^{65}$ Consequently, the combination of EDP plus mitotane is considered the reference regimen when a more aggressive therapeutic approach is required. Furthermore, EDP plus mitotane is the only treatment approach in ACC whose results have been successfully confirmed in a randomised trial. ${ }^{65}$ 


\section{Conclusions}

ACC is a disease characterised by high mortality. Surgery represents the only feasible therapy while mitotane is a drug approved for the medical treatment. However, its effectiveness as adjuvant therapy is still debated, in particular because data are retrospectively collected and therefore might be methodologically biased. The recent $\mathrm{ESMO}^{33}$ and $\mathrm{NCCN}^{34}$ guidelines recommend mitotane use in an adjuvant setting, after surgery, in patients with incomplete resection or high-risk degree. In advanced disease, a regimen with EDP in combination with mitotane is recommended. The major difficulty in the management of mitotane treatment is related to side effects and to the risk of toxicity, which requires careful and frequent monitoring of plasmatic mitotane levels. It is irrefutable that ACC requires alternative medical strategies. In the past few decades, better molecular characterisation of ACC has been tempted. ${ }^{9}$ In addition to activation of the beta catenin pathway, other findings include TP53 mutations found in Li-Fraumeni syndrome, the APC and CTNNB1 genes mutations in familial adenomatous polyposis coli, and the CDKN1C and IGF-2 genes mutations in Beckwith-Wiedemann syndrome ${ }^{7}$ as well as NF1, RB1 and menin gene mutations. ${ }^{66}$ However, even if these discoveries represent important findings in understanding the molecular changes in ACC, targeting these pathways currently does not seem to have an impact on the course of the disease. ${ }^{67}$ Waiting for new medical strategies on molecular targets in the coming years will be mandatory to optimise the current knowledge by prospective trials, to evaluate the possibility of radiotherapy in particular settings and to validate the use of mitotane in the adjuvant setting, as proposed by ADIUVO study. Importantly, owing to the rarity of the disease, collaborative studies appear to be the only way to improve the knowledge of the disease, survival and quality of life of patients with ACC. $\square$
1. Kerkhofs TM, Verhoeven RH, Van der Zwan JM, et al. Adrenocortical carcinoma: a population-based study on incidence and survival in. a Eur $J$ Cancer. 2013:49:2579-86.

2. Else T, Kim AC, Sabolch A, et al. Adrenocortical carcinoma. Endocr Rev. 2014;35:282-326.

3. Icard P, Goudet P, Charpenay C, et al. Adrenocortical carcinomas: surgical trends and results of a 253-patient series from the French Association of Endocrine Surgeons study group. World I Surg. 2001:25:891-7.

4. Pommier RF, Brennan MF. An eleven-year experience with adrenocortical carcinoma. Surgery 1992:112:963-70.

5. Schulick RD, Brennan MF. Long-term survival after complete resection and repeat resection in patients with adrenocortical carcinoma. Ann Surg Oncol. 1999;6:719-26.

6. Fassnacht M, Johanssen S, Quinkler M, et al. Limited prognostic value of the 2004 International Union Against Cancer staging classification for adrenocortical carcinoma: proposal for a Revised TNM Classification. Cancer. 2009;115:243-50.

7. Ayala-Ramirez M, Jasim S, Feng L, et al. Adrenocortical carcinoma: clinical outcomes and prognosis of 330 patients at a tertiary care center. Eur J Endocrinol. 2013;169:891-9.

8. Schteingart DE, Doherty GM, Gauger PG, et al. Management of patients with adrenal cancer: recommendations of an international consensus conference. Endocr Relat Cancer. 2005; 12:667-80.

9. Waszut U, Szyszka P, Dworakowska D. Understanding mitotane mode of action. J Physiol Pharmacol. 2017;68:13-26.

10. Kerkhofs TM, Ettaieb MH, Hermsen IG, et al. Developing treatment for adrenocortical carcinoma. Endocr Relat Cancer. 2015;22:R325-38.

11. Hart MM, Reagan RL, Adamson RH. The effect of isomers of DDD on the ACTH-induced steroid output, histology and ultrastructure of the dog adrenal cortex. Toxicol App/ Pharmacol. 1973;24:101-13.

12. Reid $L E$, Behrend $E N$, Martin $L G$, et al. Effect of trilostane and mitotane on aldosterone secretory reserve in dogs with and mitotane on aldosterone secretory reserve in dogs with
pituitary-dependent hyperadrenocorticism. J Vet Intern Med. pituitary-dependent

13. Kintzer PP, Peterson ME. Mitotane $\left(0, p^{\prime}-D D D\right)$ treatment of 200 dogs with pituitary-dependent hyperadrenocorticism J vet Intern Med. 1991:5:182-90

14. Hescot $S$, Amazit L, Lhomme M, et al Identifying mitotaneinduced mitochondria-associated membranes dysfunctions: metabolomic and lipidomic approaches. Oncotarget. 2017;8:109924-40

15. van Slooten $\mathrm{H}$, Moolenaar AJ, van Seters AP, et al. The treatmen of adrenocortical carcinoma with o,p'-DDD: prognostic implications of serum level monitoring. Eur J Cancer Clin Oncol. 1984;20:47-53.

16. Lehmann TP, Wrzesinski T, Jagodzinski PP. The effect of mitotane on viability, steroidogenesis and gene expression in $\mathrm{NCIH} 295 \mathrm{R}$ adrenocortical cells. Mol Med Rep. 2013;7:893-900.

17. Chortis $V$, Taylor $A E$, Schneider P, et al. Mitotane therapy in adrenocortical cancer induces CYP3A4 and inhibits 5alphareductase, explaining the need for personalized glucocorticoid and androgen replacement. J Clin Endocrinol Metab. 2013;98:161-71.

18. Stacpoole PW, Varnado CE, Island DP. Stimulation of rat liver 3-hydroxy-3-methylglutaryl-coenzyme A reductase activity by o, $p^{\prime}$-DDD. Biochem Pharmacol. 1982;31:857-60.

19. Maher VM, Trainer PJ, Scoppola A, et al. Possible mechanism and treatment of $0, p^{\prime} \mathrm{DDD}$-induced hypercholesterolaemia. Q J Med. 1992;84:671-9.

20. Bradlow HL, Fukushima DK, Zumoff B, et al. A peripheral action of $\mathrm{O}, \mathrm{P}^{\prime}$-Ddd on steroid biotransformation. J Clin Endocrinol Metab. 1963:23:918-22.

21. Brown RD, Nicholson WE, Chick WT, et al. Effect of o,p'DDD on human adrenal steroid 11 beta-hydroxylation activity. I Clin Endocrinol Metab. 1973:36:730-3.

22. Sbiera S, Leich $E$, Liebisch $G$, et al. Mitotane inhibits Sterol$\mathrm{O}$-Acyl transferase 1 triggering lipid-mediated endoplasmic reticulum stress and apoptosis in adrenocortical carcinoma cells. Endocrinology. 2015:156:3895-908.

23. van Seters AP, Moolenaar AJ. Mitotane increases the blood levels of hormone-binding proteins. Acta Endocrinol (Copenh). 1991;124:526-33.
24. Nader N, Raverot G, Emptoz-Bonneton A, et al. Mitotane has an estrogenic effect on sex hormone-binding globulin and corticosteroid-binding globulin in humans. I Clin Endocrinol Metab. 2006;91:2165-70.

25. Kroiss M, Quinkler M, Lutz WK, et al. Drug interactions with mitotane by induction of CYP3A4 metabolism in the clinical management of adrenocortical carcinoma. Clin Endocrinol (Oxf). 2011:75:585-91.

26. Cuddy PG, Loftus LS. Influence of mitotane on the hypoprothrombinemic effect of warfarin. South Med $\mathrm{J}$. 1986:79:387-8.

27. Stigliano A, Cerquetti L, Lardo $P$, et al. New insights and future perspectives in the therapeutic strategy of adrenocortica carcinoma (Review). Oncol Rep. 2017:37:1301-11.

28. Terzolo M, Angeli A, Fassnacht M, et al. Adjuvant mitotane treatment for adrenocortical carcinoma. $N$ Engl J Med. 2007;356:2372-80

29. Else T, Williams AR, Sabolch A, et al. Adjuvant therapies and patient and tumor characteristics associated with survival of adult patients with adrenocortical carcinoma. J Clin Endocrinol Metab. 2014;99:455-61.

30. Reidy-Lagunes DL, Lung B, Untch BR, et al. Complete responses to mitotane in metastatic adrenocortical carcinoma-a new look at an old drug. Oncologist. 2017;22:1102-6.

31. The electronic Medicines Compendium. Lysodren $500 \mathrm{mg}$ tablets. Available at: www.medicines.org.uk/emc/product/80 (accessed 28 April 2018).

32. Epiclin. Adiuvo. Available at: www.epiclin.it/adiuvo (accessed 27 april 2018)

33. Berruti A, Baudin E, Gelderblom H, et al. Adrenal cancer: ESMO Clinical Practice Guidelines for diagnosis, treatment and followup. Ann Oncol. 2012;23 Suppl 7:vii131-8.

34. www.nccn.org/professionals/physician_gls/pdf/ neuroendocrine.pdf (accessed 29 April 2018).

35. Fassnacht M, Dekkers $\mathrm{O}$, Else T, et al. European Society of Endocrinology Clinical Practice Guidelines on the Managemen of Adrenocortical Carcinoma in Adults, in collaboration with the European Network for the Study of Adrenal Tumors. Eur J Endocrinol. 2018;pii: EJE-18-0608. doi: 10.1530/EJE-18-0608 [Epub ahead of print].

36. Baudin E, Pellegriti G, Bonnay M, et al. Impact of monitoring plasma 1,1-dichlorodiphenildichloroethane (o, $\left.\mathrm{p}^{\prime} \mathrm{DDD}\right)$ levels on the treatment of patients with adrenocortical carcinoma Cancer. 2001:92:1385-92.

37. Hermsen IG, Fassnacht M, Terzolo M, et al. Plasma concentrations of $0, p^{\prime} D D D, 0, p^{\prime} D D A$, and $0, p^{\prime} D D E$ as predictors of tumor response to mitotane in adrenocortica carcinoma: results of a retrospective ENS@T multicenter study. J Clin Endocrinol Metab. 2011;96:1844-51.

38. Terzolo M, Baudin AE, Ardito A, et al. Mitotane levels predic the outcome of patients with adrenocortical carcinoma treated adjuvantly following radical resection. Eur J Endocrinol. 2013;169:263-70

39. Megerle F, Herrmann W, Schloetelburg W, et al. Mitotane monotherapy in patients with advanced adrenocortical carcinoma. J Clin Endocrinol Metab. 2018:103:1686-95.

40. Terzolo M, Pia A, Berruti A, et al. Low-dose monitored mitotane treatment achieves the therapeutic range with manageable side effects in patients with adrenocortical cancer. J Clin Endocrinol Metab. 2000;85:2234-8

41. Sparagana M. Primary hypogonadism associated with $0, p^{\prime}$ DDD (mitotane) therapy. J Toxicol Clin Toxicol. 1987;25:463-72.

42. Daffara F, De Francia S, Reimondo G, et al. Prospective evaluation of mitotane toxicity in adrenocortical cancer patients treated adjuvantly. Endocr Relat Cancer 2008;15:1043-53.

43. Faggiano A, Leboulleux S, Young J, et al. Rapidly progressing high o, $p^{\prime} D D D$ doses shorten the time required to reach the therapeutic threshold with an acceptable tolerance: preliminary results. Clin Endocrinol (Oxf). 2006:64:110-3.

44. HRA Pharma IYSOSAFE Service Availabe at: www.hra-pharma.com/index.php/en/our_products/ endocrinology/lysosafe_service/lysosafe_service (accessed 29 April 2018)

45. Kerkhofs TM, Derijks $\amalg$, Ettaieb MH, et al. Short-term variation in plasma mitotane levels confirms the importance of trough level monitoring. Eur J Endocrinol. 2014;171:677-83.

46. Neuman O, Bruckert E, Chadarevian R, et al. Hepatotoxicity of a synthetic cortisol antagonist: OP'DDD (mitotane) [article in French]. Therapie. 2001;56:793-7.

47. Baudry C, Coste J, Bou Khalil R, et al. Efficiency and tolerance of mitotane in Cushing's disease in 76 patients from a single center Eur J Endocrinol. 2012;167:473-81.

48. Pape E, Feliu C Yelehe-Okouma M, et al High-dose mitotaneinduced encephalopathy in the treatment of adrenocortical carcinoma. Oncologist. 2018:23:389-90.

49. Goto T, Miyako K, Kuromaru R, et al. Case report: adjuvant therapy with a high dose of mitotane for adrenocortical carcinoma in a 4-year-old boy. Clin Pediatr Endocrinol. 2008:17:71-4.

50. Robinson BG, Hales IB, Henniker AJ, et al. The effect of o,p'-DDD on adrenal steroid replacement therapy requirements. Clin Endocrinol. 1987;27:437-44.

51. Kupfer D, Balazs T, Buyske DA. Stimulation by o, $p^{\prime}-\mathrm{DDD}$ of cortisol metabolism in the guinea pig. Life Sciences. 1964;3:959-64.

52. Bledsoe T, Island DP, Ney RL, et al. An effect of O,P'-Ddd on the extra-adrenal metabolism of cortisol in man. J Clin Endocrino Metab. 1964;24:1303-11.

53. Weigel M, Hahner S, Sherlock M, et al. Immediate versus modified release hydrocortisone in mitotane-treated patients with adrenocortical cancer. Clin Endocrinol (Oxf). 2017;86:499-505

54. Lacroix A. Approach to the patient with adrenocortical carcinoma. J Clin Endocrinol Metab. 2010;95:4812-22.

55. Alexandraki KL, Kaltsas GA, le Roux CW, et al. Assessment of serum-free cortisol levels in patients with adrenocortical carcinoma treated with mitotane: a pilot study. Clin Endocrinol (Oxf). 2010;72:305-11.

56. Carrozza C, Lapolla R, Gervasoni J, et al. Assessment of salivary free cortisol levels by liquid chromatography with tandem mass spectrometry (LC-MS/MS) in patients treated with mitotane. Hormones (Athens). 2012:11:344-9.

57. Manenschijn L, Quinkler M, van Rossum EF. Hair cortisol measurement in mitotane-treated adrenocortical cance patients. Horm Metab Res. 2014:46:299-304.

58. Gentilin E, Tagliati F, Terzolo M, et al. Mitotane reduces human and mouse ACTH-secreting pituitary cell viability and function. Endocrinol. 2013,218:275-85.

59. Reimondo G, Puglisi S, Zaggia B, et al. Effects of mitotane on the hypothalamic-pituitary-adrenal axis in patients with adrenocortical carcinoma. Eur J Endocrinol. 2017:177:361-7

60. Zatelli MC, Gentilin E, Daffara F, et al. Therapeutic concentrations of mitotane $\left(0, p^{\prime}-\mathrm{DDD}\right)$ inhibit thyrotroph cell viability and TSH expression and secretion in a mouse cell line model. Endocrinology. 2010;151:2453-61.

61. Schteingart DE. Conventional and novel strategies in the treatment of adrenocortical cancer. Braz J Med Biol Res. 2000;33:1197-200.

62. Bates SE, Shieh CY, Mickley LA, et al. Mitotane enhances cytotoxicity of chemotherapy in cell lines expressing a multidrug resistance gene (mdr-1/P-glycoprotein) which is also expressed by adrenocortical carcinomas. J Clin Endocrinol Metab. 1991;73:18-29.

63. Khan TS, Imam H, Juhlin C, et al. Streptozocin and o,p'DDD in the treatment of adrenocortical cancer patients: long-term survival in its adjuvant use. Ann Oncol. 2000;11:1281-7.

64. Berruti A, Terzolo M, Sperone P, et al. Etoposide, doxorubicin and cisplatin plus mitotane in the treatment of advanced adrenocortical carcinoma: a large prospective phase II trial. adrenocortical carcinoma. a large prospect

65. Fassnacht M, Terzolo M, Allolio B, et al. Combination chemotherapy in advanced adrenocortical carcinoma. N Eng/ J Med. 2012;366:2189-97.

66. Zheng $\mathrm{S}$, Cherniack AD, Dewal $\mathrm{N}$, et al. Comprehensive pan-genomic characterization of adrenocortical carcinoma. Cancer Cell. 2016;29:723-36.

67. Varghese J, Habra MA. Update on adrenocortical carcinoma management and future directions. Curr Opin Endocrino Diabetes Obes. 2017;24:208-14. 